\title{
Enhancing development trajectories of synthetic environments
}

\author{
Roy Damgrave*, Eric Lutters (1) \\ Department of Design, Production and Management, Faculty of Engineering Technology, University of Twente, Enschede, The Netherlands
}

A R T I C L E I N F O

Keywords:

Virtual reality

Decision making

Synthetic environments

\begin{abstract}
A B S T R A C T
This research presents a framework that supports all stakeholders in the development of a Synthetic Environment. Guidance and support are provided throughout the entire process of development. Multiple disciplines are involved in this process, and the communication and collaboration between them is facilitated in such a way that mutual understanding is enhanced. Moreover, the rationale of decisions made throughout the development can be documented and accessed in such a way that all stakeholders can review and comprehend these decisions in relation to the prior and underlying decision-making processes.
\end{abstract}

(C) 2018 Published by Elsevier Ltd on behalf of CIRP.

\section{Introduction}

Synthetic Environments (SE) are design environments that bring together real and virtual components to allow for adequately experiencing shared information [1]. They range from small setups, representing e.g. working with a new machine, to large systems for the conjoint development of an aircraft interior. Synthetic Environments are composed out of a wide variety of tools, techniques, hardware and software components. To ensure that an SE meets all functional specifications and requirements with adequate quality, the process to configure an SE requires structure, vigour and predictability, but also flexibility and adaptability. At the same time, many different stakeholders are involved in the creation of an SE, ranging from engineers via marketers and maintenance staff to end users.

Establishing and employing SEs is often hampered by a lack of insight in the consequences of implementation. Moreover, during implementation of an SE, many stakeholders cannot yet signify or formulate how the SE could have the most relevance for them.

The research described here provides an approach for SE development, comprising the entire SE life cycle and spans multiple levels of aggregation (i.e. strategic, tactical, operational). Obviously, multiple stakeholders and multiple perspectives need to be involved in such a way that mutual understanding is enhanced [2]. This is facilitated by enabling communication and collaboration, but foremost by maintaining an overview of the configuration process that underlies the momentary status of the SE development. Also, design rationale is captured, so all stakeholders can review and comprehend decisions as well as the preceding and underlying decision-making processes.

The proposed framework renders the development cycles of SEs more predictable and make the SEs more adaptable and better configurable. Additionally, the resulting SEs will be better tailored to

\footnotetext{
* Corresponding author.

E-mail address: r.g.j.damgrave@utwente.nl (R. Damgrave).
}

the perspectives of all stakeholders. The framework should not dictate or predefine the SE development process, but should function as a tool for enhancing this process. Furthermore, the framework should be able to reuse and build on the developed methods and processes from earlier and other development or research projects.

\section{Scope and aim}

The development of an SE is, essentially, a typical design trajectory, including all the variety in stakeholders, perspectives and aspects that play a role in development cycles for products or product-service systems [3-5]. This renders many design methods and methodologies valid, but these are not self-evidently effective for, or tailored to, the specifics of developing an SE. These specifics relate to, for example, the extreme configurability of SEs, the SE being a combination of a design/ development/configuration effort and the potential re-usability of (components of) an SE and the knowledge thereof [6]. At the same time, quite some experience is available on implementing SEs [6]. By combining such domain knowledge with a design strategy that does justice to all stakeholders/perspectives involved, a design approach for SEs can be devised that effectively and efficiently facilitates SE developers in a structured and predictable manner.

\subsection{Outline of the approach}

One of the major risks in developing an SE are misalignments between stakeholders. Also, stakeholders relying on incomplete, unreliable, misinterpreted or uncertain information can hamper or endanger the development cycle. Thus, having one collective reference or starting point is pivotal in SE development. Actually, it is even more important than in product development, as an SE usually is merely a temporal cumulation of its constituents, subsequently or simultaneously serving stakeholders with different intents and aims. Moreover, some end-users will only occasionally interact with an SE, some may be responsible for building, adapting or maintaining SEs on a professional basis and 
others may be suppliers of hardware, models or methods. A support framework is introduced to provide all stakeholders with a common basis. This framework is the reference for all interactions, information/knowledge exchange and coordination activities in SE development. To allow for such functionality, the framework should offer a shared information repository throughout all development phases. For purposeful (re-)use of the information content, the framework should also provide insight in the network of relations and dependencies that connect initial stakeholder requirements to the eventual elements that together shape the Synthetic Environment.

In a sense, this depiction of the framework for SE development presents it as a kind of market-place, where negotiations and trade-off take place, in the context of both the external limitations/constraints as well as the set aimand rationale of theSE underdevelopment.Here, also the relevance of the (subjective) preferences of individual stakeholders come to fruition. This market-place metaphor might do justice to the flexibility and adaptability that is required in the process, but the structure, vigour and predictability of the approach might not thrive on it. Therefore, the framework should be able to render an overview of the design freedom that the stakeholders (still) have in de development cycle. This so-called 'solution space' is the interpretation and instantiation of the initial requirement specification of the SE, while also representing the boundaries, (subjective) preferences, decisions and considerations, as set by all stakeholders involved. The solution space also addresses all external factors that have been captured.

It goes without saying that no SE is the only possible option to achieve a certain goal. Therefore, any solution space for an SE is an instantiation of a possible solution. With this, the solution space is related to a 'discussion space', which captures an overview of these possible solutions. As such, the discussion space is the overlap of all the input by the stakeholders. It captures the mutual interests, all requirements and connected information content. The origin of this is the set of stakeholders involved, each providing their contribution to the process. This collective contribution is referred to as the 'scouting space', consisting of a 'blueprint' for every stakeholder.

With this, the approach starts by collecting information from stakeholders by means of the blueprints. Conjointly, these form the 'scouting space', from which the discussion space is the repercussion. As an accompanist of the development activities, the solution space renders the current state of affairs. Eventually, the framework brings together all intersections into an integrated foundation for SE development.

The following section belabours the individual elements of the framework in more detail, starting with the blueprints.

\section{Components of the approach}

\subsection{Blueprint}

The first step to come to a functional SE is to provide the stakeholders with means to gather and document their requirements on the SE. For this purpose, a blueprint aids a stakeholder to connect his goals and context to the (desired or envisaged) functionality of the SE. Fig. 1 gives an overview of such a blueprint, where the main elements of the SE are addressed. The current portfolio of nine elements is based on theoretical research and practical experience. Each element in the blueprint is an information container for documenting relevant information from the perspective of one stakeholder [7]. With this, the stakeholder is provided simultaneously with a 'backbone', a 'checklist' and a 'notebook' to express requirements. Thus, the stakeholder has a structured manner to work from the core of Fig. 1, capturing the intent or stated purpose [1] towards the formulation of his functional contribution to the SE.

Obviously, the experience gained in setting up an SE can be effectively reused in other projects. For this reason, the structure of a blueprint can be updated as more SEs are build. Moreover, new insights and increased knowledge can be used to update blueprint structures. This yields a set of template blueprints that are effectively

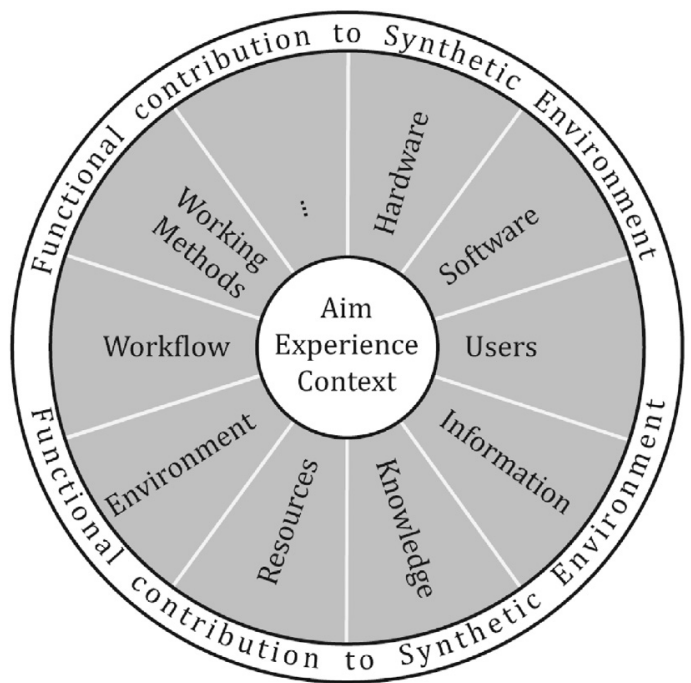

Fig. 1. Template blueprint with main elements.

tailored to the needs of specific types of stakeholders. This not only increases the efficiency of capturing the stakeholders' viewpoints, but it also facilitates stakeholders in maintaining an overview of their own perspectives as concerns completeness and focus.

An additional advantage of using template blueprints is that the input of all stakeholders can be integrated in a structured and reproducible manner. In this, the information provided by stakeholders working at different levels of aggregation, completeness, certainty and reliability can be brought together, without any judgement on priority and sequence. As the blueprints provide a taxonomy of elements that are expected to contribute to the discussion space (Section 3.3). Therefore, there is no need for a blueprint to enforce or pre-determine the demarcation or scope of the requirements that are formulated. The amount of documentation related to the different elements can vary, according to the interests, priorities and expertise of the stakeholders. Given the character of, and idea behind the blueprint, its elements are foremost ways to cluster the requirements [8] rather than a prescribed subdivision. Any blueprint template will therefore be a facilitator rather than a standardised straitjacket. Moreover, all elements can be used to express information on the different levels of aggregation that the stakeholders may be interested in, from strategic (e.g. provider of the $\mathrm{SE}$ ), via tactical (e.g. operator configuring the SE) to operational (e.g. client that will use the envisaged SE).

\subsection{Scouting space}

Individual blueprints will not allow for purposeful decisions or constructive alignment in the development cycle of the SE. An additional layer is required to process the blueprints, while also suggesting viable solutions paths to arrive at an adequate SE. Interrelating blueprints will interrelate stakeholders, and will ensure that common concerns or potentialities emerge. The aggregated blueprints establish a convex hull that delineates the scope and interest of the joint stakeholders. This convex hull is defined as the scouting space of the SE development cycle.

As the size of a scouting space increases (for larger and complex SE developments with many stakeholders), it is an excellent means to distinguish overlaps and contradictions amongst stakeholders. Comparing scouting spaces amongst projects, also indicates voids and potential omissions. Moreover, the scouting spaces allows stakeholders to empathise with other viewpoints, and with considerations at other levels of aggregation. The blueprints will thus act as a base and trigger for discussion, in which the information content serves as a structured foundation.

The scouting space is not a sequel to the use of the blueprints, it rather is a dynamic representation thereof, allowing stakeholders 


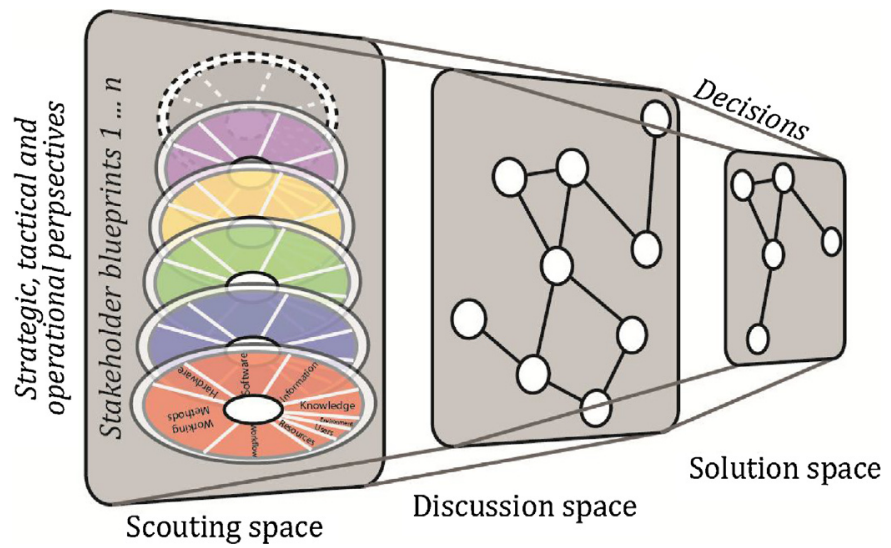

Fig. 2. Overview of the relation between blueprints, scouting space, discussion space and solution space.

to continuously interact with the blueprints. The way in which they express their intents and requirements, as well as the way in which they prioritise elements and interrelate functionality of the SE leads to meta-data on the SE development. By adding this metadata, e.g. by tagging requirements, the information from the requirement repository can be adequately filtered in view of the circumstances later in the development cycle. This filtering of information prevents an information overload $[3,9]$ if similarities between different blueprints require attention, or if the impact of requirements on a specific viewpoint or element should be communicated amongst stakeholders.

The scouting space is the means to capture the SE significance for the stakeholder and makes priorities and concerns visible, while exposing voids, uncertainties and ambiguities. Thus, the scouting space gives an unprepossessed and evenhanded starting point for the actual construction of the SE (Fig. 2).

\subsection{Discussion space}

The scouting space captures the potential of the solution without any bias, so the next layer aims to tackle deliberations and forethoughts on the joint requirements that represent the maximum freedom for the SE development. The convex hull of the scouting space is the basis for the discussion space, establishing the base and outer-boundaries for the development of the SE. This discussion space relates all information from the blueprints from the stakeholders, and can be visualised as a graph. The nodes in the graph originate from the requirements documented in the blueprints. The edges in the graph stem from the amalgamation of the blueprints, but also represent the metadata that was added to the requirements by means of e.g. the tags. With this, the edges structurally link entities, but they also carry denotation on different reasons for linking the nodes. Therefore, the discussion space can be regarded as a conceptual graph.

\subsection{Solution space}

From this discussion space, a more consolidated space emerges, containing the essential information to construct the SE. This is the solution space (Fig. 2), as a converging evolvement of the discussion space. During development, decisions are provoked by (changes in) the discussion space and decisions are reached that change nodes or edges in the conceptual graph of the discussion space. The solution space needs to reflect the consequences of such decisions and is subjective by definition, as it captures the repercussions of compromises and considerations. Also, the solution space caters for the creative and non-deterministic steps that qualify the development of an SE as a design activity. Nevertheless, the solution space will maintain the structured provision of information that is essential throughout the entire development cycle.

In terms of the conceptual graph of the discussion space, the solution space is foremost a subgraph, thus capturing the converging course of processes. Thereupon, this subgraph will contain less nodes and edges, as it is a consolidated rendering of the discussion space. All kinds of variants, void thinking patterns and trials are left out, and only underpinned decisions will be used as stepping stones for further development, guided by strategical, tactical as well as operational considerations. These play an important role, as the triad scouting-discussion-solution space has a split focus: firstly, on the (combinability of) requirements and technical feasibility of potential solutions; secondly, on the management of the project that implements the SE. The latter implies that the solution space also add (subjective) constraints as regards for example:

- Envisaged maturity level, or market-readiness

- Capacity available at the moment, or for this purpose

- Capability at the moment, or for this purpose

- Company strategy or company policy

- Financial criteria or financial scope

- Logistics or lead time

- Investments in stakeholder relations or stakeholder buy-in

\section{Framework}

Combining blueprints, scouting space, discussion space and solution space layers, expresses the potential of the SE. Fig. 3 illustrates the overall framework for this, denoting the handling of requirements on the left side and the instantiated SE on the right side. The instantiation contains the configured tools, techniques, hardware and software components that are the result of the development cycle. There is no sequential relation between the parts of the framework. For example, scouting new requirements may be the result of changes in an instantiated SE, the addition of a stakeholder can significantly influence the discussion space and an observed void in the discussion space could both trigger a request for the update of a blueprint or might cause a different way of implementing an instantiated SE.

The framework facilitates communication, collaboration and discussion among stakeholders. It does not impose any process, it rather provides flexible support of all activities related to developing an SE. It gives insight in the different perspectives of the solution and offers a documentation approach.

The framework provides multiple views on the same targeted $\mathrm{SE}$, at different levels of aggregation. Based on the information repository, this enables a purposeful formulation of the design rationale. With this, individual stakeholders are facilitated in initiating, substantiating and assessing the evolvement of the SE development, embedded in the context and requirement specification of all stakeholders [10].

Establishing SEs based on the framework allows for structured working methods as well as for predictable and reliable outcomes.

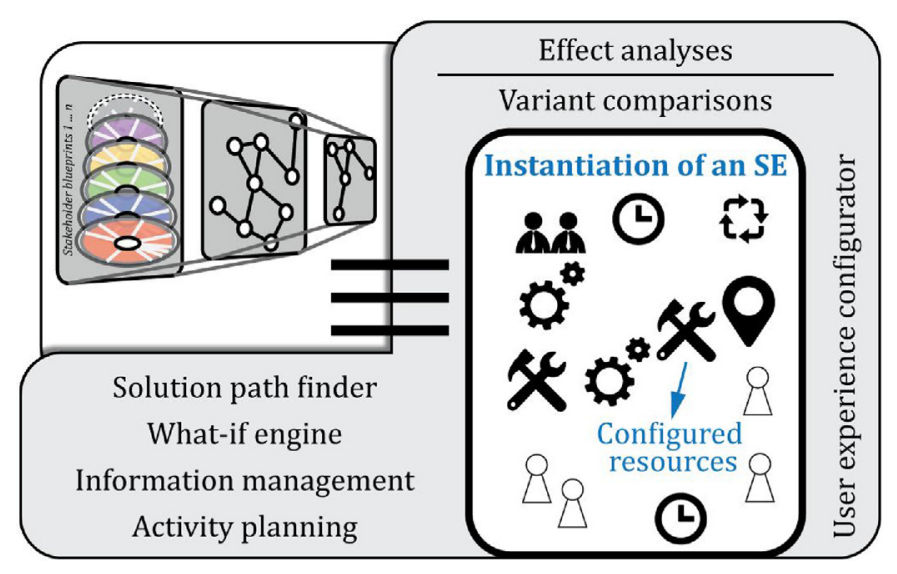

Fig. 3. Framework for SE development. 
Moreover, because of the structured and explicit graph-based representation of the information content, it becomes feasible to mutually compare SEs and to compare and weigh up design variants for SEs under development. However, the most relevant advantage is that development/adaptation of SEs can now heavily rely on the reuse of all expertise that is captured explicitly and accessibly. This renders development cycles for SEs much more effective and efficient. Moreover, the information that is gained in building SEs contributes to more and more profound knowledge on establishing and using SEs. Therefore, with increased use of the framework, SE development can progressively start with quickly configuring a substantiated skeleton for the SE by reusing existing modules. Obviously, this significantly increases the predictability of SE development; even partial automation comes within reach. Also, the framework may pro-actively suggest relevant (configurations of) solutions, including indications for e.g. feasibility, suitability and timeliness. With this, for SE developers the focus can be on the finesses of optimising the SE instead of on redeveloping new SEs every time.

To allow for flexible and adaptable facilitation of SE development, the framework also supports 'what-if' questions. It, for example, allows for the simulation of the consequences of design decisions as well as for roll-back approaches that 'undo' design decisions while still learning from them. Thus, single or multiple steps back in the process can be made to correct an error or to try another possible approach without having detriment on the information content. Ultimately, this gives the opportunity to continue optimising existing SEs. To achieve the most appropriate SE solution, the solutions applied need to be based on the tools that are available and accessible at the time of implementation. At any given moment, new tools and techniques do come to the market, which may influence the composition of the solution. This should not be a surprise or threat, but an opportunity to constantly improve the solution. If a new tool becomes available, it can be examined within which current SEs it can be applied in order to increase efficiency or effectiveness and at what consequences in terms of quality, cost and time.

\section{Exploratory cases}

In the Virtual Reality \& Smart Industry Lab of the University of Twente, the framework is the main driver for SE development. A practical exemplary case performed in this facility is supporting the design of a factory. In this situation all design information was created and scattered among five specialized engineering agencies. A SE was developed to combine this information in one virtual environment, enabling to review the integration of, and the relation between, the different drawings. The visualized potential factory could be assessed (simultaneously) from different perspectives, aligned with the stakeholders. First a constructive view to understand how individual design decisions influence each other, secondly a combined managerial overview to review the exposure of the factory and integration with existing assets, and third a future user study where factory employees could already perform their future task in a virtual environment. In the process of the development of the SE the already available data was treasured, and individual engineers were not forced to change their working method to adhere to the SE; the SE was adopted to work with the unprocessed data from different sources. The advantages of reusing elements from existing SEs or from other teams were visible, however, the most relevant observation was that the execution of the development cycles did indeed significantly contribute to the information content and the elaboration of e.g. the building blocks and re-use libraries.

The practical case studies that have been executed show the feasibility of the approach; however, they do not fully exploit the possibilities and opportunities provided by the framework. For example, interpreting aspects on re-using information, crossfertilisation between projects and increased efficiency in configuring SE requires a substantial portfolio of sufficient and meaningful projects. Such projects become more relevant as more stakeholders and perspectives are involved, which implies lead times of multiple years. Currently, a number of large SE development projects are being supported by the framework and the experience that already has been captured. In these projects, focus is on adequately guiding and governing the projects, as well as on purposefully strengthening the framework and its content based on the learnings from the projects. An example of such a larger project in which the framework is used encompasses the development, building, commissioning and operation of a number of physical pilot plants for advanced manufacturing, addressing the entire spectrum from business models, via simulating innovative production processes and machine tools to quality control, logistics and building management.

\section{Concluding remarks}

Experiencing and interacting with shared information in development cycles is key to the use of SEs. Up to now, building SEs all too often relies on artisanship and eventualities, with a lot of implicit path dependencies. This leads to a dichotomy between new technology and traditional skills. The explorations and implementations described in this research depict the current insights in an ongoing process towards a robust, transparent and flexible development approach for SE development. This is supported by a framework that in itself co-develops as more experience and knowledge is acquired. At the same time, case studies have already substantiated that the framework is definitely applicable and adequate for the development of SEs with various scopes, application areas and extents.

Future plans include encapsulating the framework in a business model, allowing industry to gain quick and effective access to Synthetic Environments. Research efforts will focus mostly on extending knowledge capturing as well as on expanding selflearning capabilities of the framework and autonomous generation of proposals/variants and the evaluation thereof.

\section{References}

[1] Miedema J, Van der Voort MC, Van Houten FJAM (2009) Advantageous Application of Synthetic Environments in Product Design. CIRP Journal of Manufacturing Science and Technology 1:159-164.

[2] Tomiyama T, D’Amelio V, Urbanic J, Eimaraghy W (2007) Complexity of MultiDisciplinary Design. CIRP Annals - Manufacturing Technology 56:185-188.

[3] Elmaraghy W, Elmaraghy H, Tomiyama T, Monostori L (2012) Complexity in Engineering Design and Manufacturing. CIRP Annals - Manufacturing Technology 61:793-814.

[4] Exner K, Stark R (2015) Validation of Product-Service Systems in Virtual Reality. Procedia CIRP 30:96-101.

[5] Vasantha GVA, Roy R, Lelah A, Brissaud D (2012) A Review of Product-Service Systems Design Methodologies. Journal of Engineering Design 23:635-659.

[6] Damgrave RGJ, Lutters D, Van Houten FJAM (2013) The Virtual Reality Lab as a Synthetic Environment: From Strategic Approach to Practical Implement. Smart Product Engineering 787-794

[7] Unger D, Eppinger S (2011) Improving Product Development Process Design: A Method for Managing Information Flows, Risks, and Iterations. Journal of Engineering Design 22:689-699.

[8] Lutters D, Van Houten FJAM, Bernard A, Mermoz E, Schutte CSL (2014) Tools and Techniques for Product Design. CIRP Annals - Manufacturing Technology 63:607-630.

[9] Dhuieb MA, Laroche F, Bernard A (2016) Context-Awareness: A Key Enabler for Ubiquitous Access to Manufacturing Knowledge. Procedia CIRP 41:484-489.

[10] Lundgren M, Hedlind M, Kjellberg T (2016) Model Driven Manufacturing Process Design and Managing Quality. Procedia CIRP 50:299-304. 